\title{
Occurrence of black stem on Helianthus annuus caused by Phoma macdonaldii and resistant evaluation of different sunflower varieties ${ }^{\text {ir }}$
}

\author{
Ningning Yan ${ }^{1}$, Ren $\mathrm{Na}^{2}$, Ruifang $\mathrm{Jia}^{1}$, Jian Zhang ${ }^{1}$ and Jun Zhao, \\ ${ }^{1}$ Department of Horticulture and Plant Protection, Inner Mongolia Agricultural University, Hohhot 010018, PR China \\ 2 Institute of Cereal and Oil Crops, Hebei Academy of Agricultural and Forestry Sciences, Shijiazhuang 050035, PR China
}

Received 11 December 2019 - Accepted 9 March 2020

\begin{abstract}
The first reported case of Phoma macdonaldii Boerema that caused sunflower black stem disease in China was from Xinjiang Uygur Autonomous Region in 2008. Black spindle lesions were observed on sunflower stems in the fields of Inner Mongolia region in China. The causal agents were isolated from the infected stems. Cultures on potato dextrose agar (PDA) plates were whitish with black particles underneath. Dark and pear-like pycnidia with yellowish or dirty whitish exudates were also observed on the surface of the cultures. The cultures produce primarily non-septate conidia. The cultures could infect sunflowers stems by artificial inoculation and developed symptoms consistent with those in the field. Morphological characteristics and molecular identification indicated the isolated causal agents were P. macdonaldii. Phylogenetic analysis based on ITS1, 5.8 s RNA and ITS2 region confirmed the identification. Disease resistance assessments were performed on twenty sunflower varieties and the results suggested that the resistance of seven sunflower varieties to P. macdonaldii was above the average; SC89 and Longkuizal were susceptible to P. macdonaldii.
\end{abstract}

Keywords: sunflower black stem / Phoma macdonaldii / resistance evaluation

Résumé - Fréquence d'un noircissement de la tige sur Helianthus annuus causée par Phoma macdonaldii et évaluation de la résistance de différentes variétés de tournesol. Le premier cas rapporté de Phoma macdonaldii Boerema induisant un noircissement de la tige du tournesol en Chine a été signalé dans la région autonome ouïgoure du Xinjiang en 2008. Des sillons de lésions noires ont été observées sur des tiges de tournesol dans les champs de la région de Mongolie intérieure en Chine. Les agents causaux ont été isolés des tiges infectées. Les cultures sur des plaques de gélose au dextrose de pomme de terre (potato dextrose agar, PDA) étaient blanchâtres avec des particules noires en dessous. Des pycnides foncées piriformes avec des exsudats jaunâtres ou blanchâtres sales ont également été observées à la surface des cultures. Les cultures produisent principalement des conidies non cloisonnées. Les cultures ont pu infecter les tiges et les feuilles de tournesol par inoculation artificielle et développer des symptômes correspondant à ceux observés sur le terrain. Les caractéristiques morphologiques et l'identification moléculaire ont indiqué que les agents causaux isolés étaient $P$. macdonaldii. L'analyse phylogénétique basée sur la région «ITS1-l'ARN 5.8s-ITS2 » a confirmé l'identification. Des évaluations de la résistance aux maladies ont été effectuées sur vingt variétés de tournesol et les résultats ont suggéré que la résistance de sept variétés de tournesol à $P$. macdonaldii était supérieure à la moyenne; SC89 et Longkuiza1 étaient sensibles à P. macdonaldii.

Mots clés : maladie de la tige noire du tournesol / Phoma macdonaldii / évaluation de la résistance

\section{Introduction}

Sunflower (Helianthus annuus) is one of the most important oil crops in the world (Darvishzadeh and Sarrafi, 2007). In China,

\footnotetext{
is Contribution to the Topical Issue "Sunflower / Tournesol".

*Correspondence: zhaojun@imau.edu.cn
}

sunflower is widely grown in Xinjiang, Gansu, Inner Mongolia, Ningxia, Shanxi, Hebei, Jilin and Heilongjiang provinces (Liu and $\mathrm{Li}, 1988$ ). Recently, with the increasing value of sunflower seeds for human diet and cooking oil, the area of sunflower cultivation has increased, and black stem disease caused by Phoma macdonaldii Boerema (teleomorph: Leptosphaeria lindquistii Frezzi) has been reported as one of the new diseases 
(Chen et al., 2008). Black stem was previously reported in Asia, Africa, North and South America and Europe (Miric et al., 1999). In China, this disease was first reported in 2008 in the Ili valley of Xinjiang Uygur Autonomous Region and 13000 hectares of sunflowers in the area were infected with P. macdonaldii, accounting for $49 \%$ of the sunflower's fields in the area (Chen et al., 2008). Sunflower cultivars JN-2519, M0314 and KWS303 are highly susceptible to $P$. macdonaldii, showing at least $44 \%$ plants infected (Chen et al., 2008). Typical symptoms of sunflower black stem caused by $P$. macdonaldii are black, round to oval lesions, centered on the leaf axil and the lesions on stems may gradually girdle the stem (Debaeke and Pérès, 2003). In this situation, symptoms of premature ripening and early senescence may occur in sunflowers, resulting in small heads and incomplete seed fill (Bordat et al., 2017). Occasionally, strong wind and rain may result in stem-breaking of sunflower at the infected sites, thus dramatically reduce yield (Bert et al., 2004).

After the first report of sunflower black stem disease in Xinjiang Uygur Region, this disease was added to the quarantine sunflower disease list at the end of 2010 in China based on the finding that seeds can be infected by P. macdonaldii and could cause long distance transmission for this disease. This regulation has contributed to preventing transmission of sunflower black stem from imported commercial seeds.

The object of this paper was to verify whether the black stem disease caused by P. macdonaldii occurred in other regions of China since its first report in Xinjiang Uygur Region in 2008. Samples of infected sunflower plants with black stem-like symptom were collected from different counties of Inner Mongolia region in 2010. The causal agent labeled as PM1, PM2 and PM3 representing the diseased samples which were collected from Dalateqi, Tumoteyouqi and Wulateqianqi, respectively. We observed morphological characteristics and tested pathogenicity. The internal transcribed spacer (ITS) sequences were also used to confirm the identifications at the molecular level. In addition, resistance evaluation was performed on different sunflower varieties under laboratory conditions.

\section{Materials and methods}

\subsection{Isolation of fungi}

In the period of August-October 2010, diseased sunflower samples were collected from different counties of Inner Mongolia region (Dalateqi, Tumoteyouqi and Wulateqianqi). Symptomatic stem fragments (Fig. 1A) were cut into $1 \mathrm{~cm}^{2}$ pieces and sterilized in $70 \%$ ethanol for $10 \mathrm{~s}$ and $3 \%$ sodium hypochlorite solution for $10 \mathrm{~min}$. The pieces were rinsed in sterile distilled water three times and dried with sterilized filter paper. Tissues were transferred onto 9 - $\mathrm{cm}$-diameter petri dishes containing PDA and incubated at $25^{\circ} \mathrm{C}$ in the dark. Three samples were collected from each location. One isolate from each location was selected to continue with the morphological, pathogenic and phylogenetic determination.

\subsection{Fungi morphology}

Isolates were subcultured on PDA at $25^{\circ} \mathrm{C}$. Mycelia, pycnidia and conidia were observed under the microscope (OLYMPUS BX41). Conidia germination was determined by

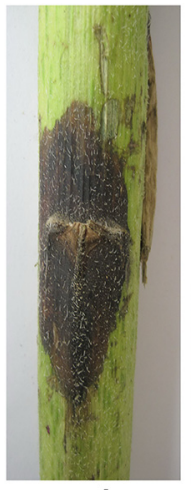

A

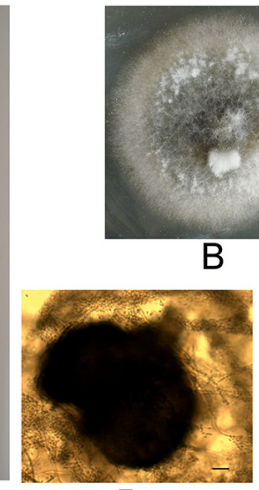

D

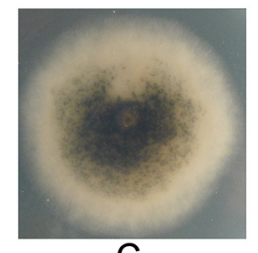

C

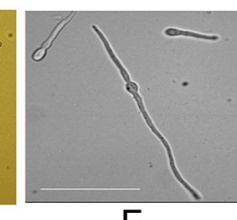

$\mathrm{E}$
Fig. 1. Symptoms of sunflower black stem and characterization of P. macdonaldii. A. Natural infection of black stem on sunflower stem. B. Culture characteristics of P. macdonaldii on PDA. Cotton-like aerial mycelia with black pycnidia underneath the central area. C. Back of the colony. D. Dark, pear-like pycnidia were observed under the microscope. E. Conidia. F. Conidia germinating from one and both sides of conidia. Scale bar $=30 \mu \mathrm{m}(\mathrm{D}, \mathrm{E})$ or $20 \mu \mathrm{m}(\mathrm{F})$.

placing a suspension of $20 \mu \mathrm{l}$ on a concave glass slide and transferring it into a microchamber and covering. The microchamber was maintained at $100 \%$ humidity by adding sterile distilled water. Conidia were incubated for 7 days before germination (Roustaee et al., 2000).

\subsection{Pathogenicity test}

Plugs with $4 \mathrm{~mm}$ diameter were cut from the actively growing edge of one-week-old colonies of each isolate and were inoculated on the sunflower stems wounded with sterile toothpick (cultivar LD5009). Plain PDA plugs were inoculated on stems as control. Each isolate was inoculated on five sunflower seedling stems as replications. To promote pathogen development, cotton absorbed with sterile distilled water was placed over the plugs, and wrapped with parafilm to fix plugs and keep humidity. After two weeks, the inoculated stems with typical symptoms were used for re-isolation.

\subsection{Fungi molecular characters}

Genomic DNA was extracted from fresh mycelia. Extraction buffer $(100 \mathrm{mM}$ Tris-HCl pH8.0; $20 \mathrm{mM}$ EDTA$\mathrm{Na}_{2} ; 1.4 \mathrm{M} \mathrm{NaCl} ; 2 \%$ Cetyltrimethyl Ammonium Bromide) was added to the well-ground mycelia pretreated with liquid nitrogen and incubated at $65^{\circ} \mathrm{C}$ for $30 \mathrm{~min}$ and centrifuged at $12000 \mathrm{r} / \mathrm{min}$ for $10 \mathrm{~min}$. Supernatant was transferred into a new tube and mixed with the same amount of phenolchloroform-isoamyl alcohol $(25: 24: 1)$ and centrifuged at $12000 \mathrm{r} / \mathrm{min}$ for $10 \mathrm{~min}$. Supernatant was transferred into a new tube and mixed with the same amount of isopropanol and centrifuged at $12000 \mathrm{r} / \mathrm{min}$ for $10 \mathrm{~min}$ for DNA precipitation. The DNA obtained was washed twice with enzyme-free water and dried for future use.

The ITS region was amplified by polymerase chain reaction (PCR) with universal primers of ITS1 and ITS4 (Xu et al., 2007). The amplified products were sequenced 
using the Sanger method (BGI Tech Solutions Co., Ltd). The region including ITS1, 5.8 $\mathrm{s}$ RNA and ITS2 was blasted in GenBank from NCBI database and aligned using Mega system (Mega 6.0). Phylogenetic analysis was performed on a dataset comprising three isolates and other Phoma species retrieved from BLAST searches.

\subsection{Plant material}

Seeds of different sunflower varieties were obtained from the Sunflower Germplasm Collection Center of Inner Mongolia Agricultural University. The seeds were sown in plastic pots $(12 \mathrm{~cm}$ diameter $\times 12 \mathrm{~cm}$ height $)$ filled with a vermiculite-clay mixture at $2 \mathrm{~cm}$ depth. Three pots were used for each variety and one pot for control, 4 seeds were planted in each pot. The pots were kept in greenhouse with $25^{\circ} \mathrm{C}$ and 14-h photoperiod for plant growing.

\subsection{Assessment of sunflower resistance}

PM3 isolated from Wulateqianqi, the main sunflower planting region in Inner Mongolia, was chosen for resistant assessment. PM3 was cultured on PDA medium at $25^{\circ} \mathrm{C}$ for 2 weeks. A conidial suspension was obtained by flooding the plates with sterile distilled water and stirring mechanically. Inoculations were made with $20 \mu \mathrm{l}\left(1 \times 10^{6}\right.$ conidia/ml $)$ of a spore suspension at the intersection of the cotyledon petioles and the hypocotyl of 4-weekold sunflower seedlings with a micropipette. Sterile distilled water was inoculated as a control. For each variety, twelve seedlings planted in three pots were inoculated and 4 plants were inoculated with distilled water as control. During the first $24 \mathrm{~h}$ post inoculation, the pots were covered with transparent bags to maintain nearly saturated humidity, favorable for fungal infection. The inoculated plants were then kept in green house with supplementary light (14-hour photoperiod at $25^{\circ} \mathrm{C}$ ). Lesion size on each inoculated stem was measured two weeks post inoculation, the average lesion size was based on the size of 12 lesions. The resistance level was classified based on the disease classification criterion (Larfeil et al., 2010) listed as follows:

- Score 1: Necrotic stem length less than $1.5 \mathrm{~cm}$ was designed as resistant;

- Score 2: Necrotic stem length between $1.5 \mathrm{~cm}$ and $3 \mathrm{~cm}$ was designed as moderate resistant;

- Score 3: Necrotic stem length between $3 \mathrm{~cm}$ and $6 \mathrm{~cm}$ was designed as susceptible;

- Score 4: Necrotic stem length greater than $6 \mathrm{~cm}$ was designed as highly susceptible.

\section{Results}

\subsection{Isolates morphology}

White, cotton-like aerial mycelia developed in a concentric zonal pattern and covered the pycnidia underneath (Fig. 1B). Abundant dark, pear-shaped pycnidia were concentrated in the center of the colony (Figs. 1C and 1D) and produced yellowish or off-white exudates. The average size of conidia for three isolates (PM1, PM2 and PM3) was between 2.5-4.0 $\mu \mathrm{m}$ (length) $\times 1-2.5 \mu \mathrm{m}$ (width) (Fig. 1E). Conidia germinated from either one-side or two-sides and formed germination

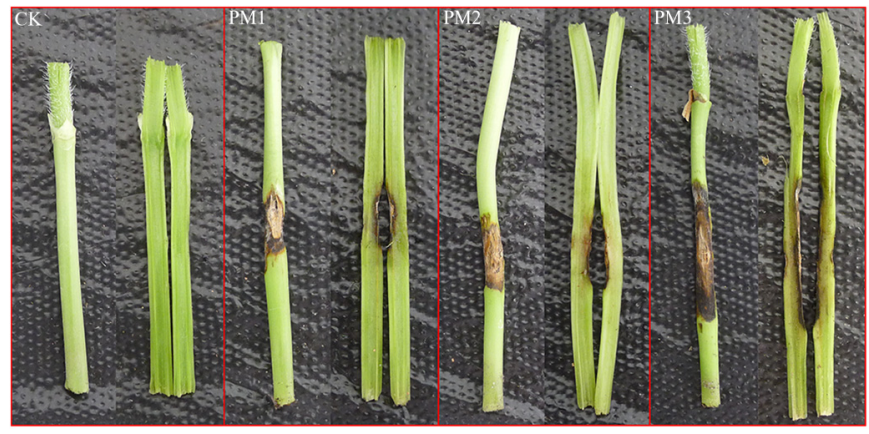

Fig. 2. The symptoms of sunflower stems post inoculated with P. macdonaldii. Sunflower stems inoculated PDA plugs with P. macdonaldii PM1, PM2 and PM3. Inoculation of plain PDA plugs was taken as a control (CK). Pictures were taken 2 weeks post inoculation. Left and right photo of each treatment indicated complete stems and longitudinal section of the corresponding stems.

tubes (Fig. 1F). The morphology of all three isolates was consistent with those of P. macdonaldii described by Boerema (Boerema et al., 2004).

\subsection{Pathogenicity test}

Sunflower stems were inoculated with PM1, PM2 and PM3. All three isolates caused black spindle lesions on the inoculated stems 14 days post inoculation (Fig. 2). Fungi were re-isolated from the junction of the healthy and diseased areas and cultured on PDA plates. Fourteen days later, the morphology of mycelium, pycnidia and conidia were similar to the original isolates, suggesting PM1, PM2 and PM3 are P. macdonaldii.

\subsection{Phylogenetic analysis of ITS}

The ITS regions of PM1, PM2 and PM3 were amplified by PCR and sequenced by the Sanger method. Nucleotide sequences with an estimated length of $481 \mathrm{bp}$ including ITS1, 5.8 s RNA and ITS2 from three isolates were aligned and showed $100 \%$ identity to each other (data not shown). The consensus sequence was blasted to GenBank and the results revealed that it shared $100 \%$ similarity with P. macdonaldii (Accession No. HM003206). The blast results of other 10 P. macdonaldii isolates were retrieved from GenBank (Tab. 1) and their regions of ITS1, 5.8 s RNA and ITS2 were used to complete the phylogenetic analysis. The phylogenetic tree was constructed based on Neighbor-joining analysis, in which PM1, PM2 and PM3 shared 100\% identity with Leptosphaeria lindquistii (teleomorph of Phoma macdonaldii) strain HM003206 (Fig. 3). The sequences of isolates PM1, PM2 and PM3 were deposited into GenBank with Accession No. KP197059, KP197060, KP197061 respectively. These results further proved that all three isolates are P. macdonaldii.

\subsection{Assessment of sunflower resistance}

To evaluate resistance of sunflower varieties to P. macdonaldii, twenty varieties were inoculated with isolate PM3. According to the resistance classification criterion 
Table 1. Isolates information used for phylogenetic analysis.

\begin{tabular}{llll}
\hline Isolate & GenBank accession number & Host plant & Geographic origin \\
\hline Leptosphaeria lindquistii & HM003206 & Helianthus annuus & China \\
Leptosphaeria lindquistii & HQ700313 & Helianthus annuus & China \\
Leptosphaeria lindquistii & AB690462 & Helianthus annuus & China \\
Leptosphaeria lindquistii & AB690463 & Helianthus annuus & China \\
Leptosphaeria lindquistii & JF740232 & Helianthus annuus & Germany \\
Leptosphaeria lindquistii & JF740233 & Helianthus annuus & Canada \\
Plenodomus confertus & KF887049 & Ferula & China \\
Leptosphaeria biglobosa & DQ458906 & Raphanus raphanistrum & Australia \\
Leptosphaeria biglobosa & DQ133893 & Brassica napus & GK \\
Leptosphaeria maculans & JX648199 & Brassica napus & Germany \\
\hline
\end{tabular}

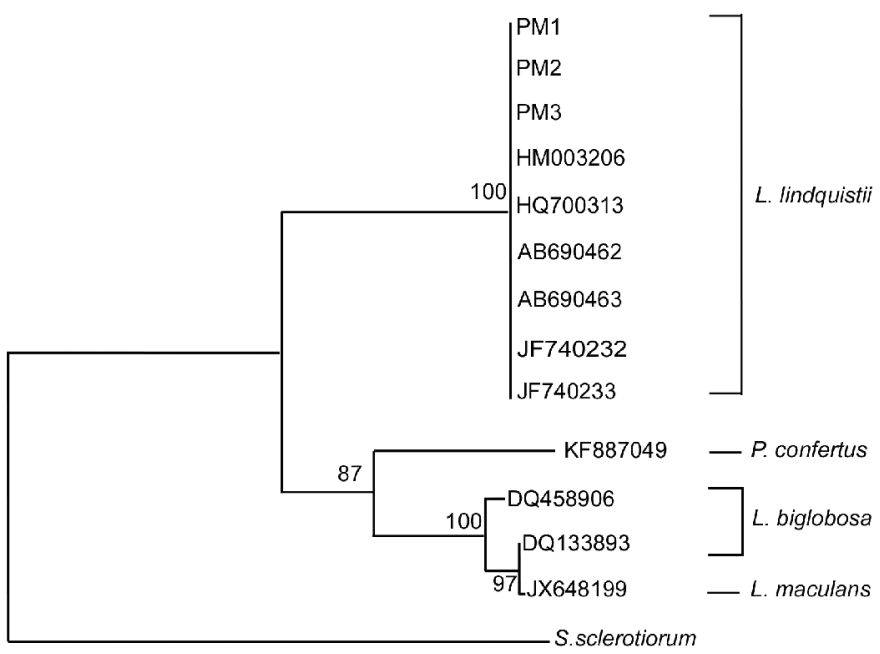

Fig. 3. Genetic relationships between three new $P$. macdonaldii isolates PM1, PM2, PM3 and related strains retrieved from GenBank. Neighborjoining tree was obtained based on the phylogenetic analysis of the ITS region including ITS1, $5.8 \mathrm{~s}$ and ITS2 by using MEGA 6.0. Ten sequences of related strains were retrieved by Blast searches. Bootstrap values above $50 \%$ were indicated (1000 replicates). The ascomycete fungi Sclerotinia sclerotiorum was used as the outgroup strain.

(Larfeil et al., 2010), Xinshikui6, CY101, Longshikui3, 118, KJ003, 7K512 and T33 with lesion length of 0.7 to $1.3 \mathrm{~cm}$, thus they were classified as resistant varieties; F60, Yikuiza3, Xinkuiza5, RH3146, 6009, Chikui 2, JK518, KS7, S31, 5009 and MGS with the lesion length of 1.55 to $2.47 \mathrm{~cm}$ and were classified as moderate resistant varieties; $\mathrm{SC} 8(3.1 \mathrm{~cm})$ and Longkuizal $(3.26 \mathrm{~cm})$ were classified as susceptible varieties (Fig. 4). None were identified as highly susceptible varieties.

\section{Discussion}

Black stem is a quarantine disease of sunflower in China and was first reported in the Ili valley of Xinjiang Uygur Region in 2008 (Chen et al., 2008). During 2010 and 2011, 185 diseased samples were collected from 37 commercial farms or individual households in Xinjiang, Inner Mongolia, Ningxia, Hebei and Beijing (Wu et al., 2012) and 105 samples

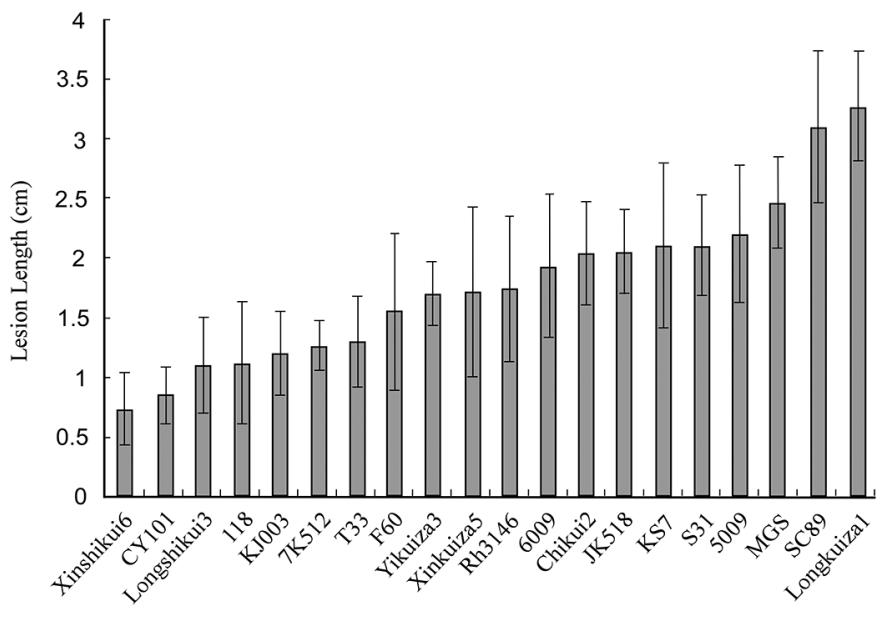

Fig. 4. Comparison the lesion lengths of different sunflower varieties post inoculated with P. macdonaldii isolate PM3. Twenty sunflowers varieties were inoculated with PM3 and the lesion lengths were measured 2 weeks post inoculation. Data represent the mean of three biological replicates and the error bars indicate standard deviation.

identified as P.macdonaldii positive were from Xinjiang, suggesting that this disease had not spread to other areas since its first discovery in Xinjiang Uygur region (Wu et al., 2012). Chinese Entry-Exit Inspection and Quarantine Bureau has imposed inspection of imported sunflower seeds for P. macdonaldii and the first intercept record was reported in 2011(Luo et al., 2011). However, based on the findings in this paper, we confirmed that black stem caused by P. macdonaldi has appeared in the Inner Mongolia region.

Even though sunflower black stem has appeared in Inner Mongolia region, the original sources of this disease in this region are still unknown. Tianjin Entry-Exit Inspection and Quarantine Bureau isolated nine strains of P. macdonaldii from sunflower seeds imported from Argentine (Luo et al., 2011). Chen and his colleagues suspected that P.macdonaldii was introduced to Xinjiang via imported commercial seeds from abroad, especially from east Europe where sunflower black stem is one of main diseases on sunflower (Chen et al., 2008). Because P. macdonaldii is a seed-borne fungus, it could be transmitted to Inner Mongolia by either international or domestic trade of sunflower seeds. 
Considering the spreading of sunflower black stem in China, it is important to evaluate the resistance level of different sunflower varieties to guide farmers to choose resistant varieties for planting. In this study, resistance evaluation was performed on 20 sunflower varieties under the laboratory condition. Varieties Xinshikui6, CY101, Longshikui3, 118, KJ003, 7K512 and T33, which showed resistance to $P$. macdonaldii, could be the best candidates for planting in areas where black stem occurs. In addition, agronomic control is also recommended to control the spread of sunflower black stem disease. Burying crop residues is very effective because there is almost no infection when infected stem pieces are buried in the soil compared with those on the soil surface (Seassau et al., 2010). Therefore, the combination of planting resistant varieties and burying debris of diseased sunflower plants could be the most effective way to control the sunflower black stem.

Acknowledgements. This work was funded by China Agricultural Research System (CARS-14), Inner Mongolia Scientific Major Project and Inner Mongolia Scientific Plan Project.

\section{References}

Bert PF, Dechamp-Guillaume G, Serre F, et al. 2004. Comparative genetic analysis of quantitative traits in sunflower (Helianthus annuus L.). Theor Appl Genet 109: 865-874.

Boerema GH, Gruyter JD, Noordeloos ME, et al. 2004. Phoma identification manual. In: G Phoma sect. Plenodomus. UK: CABI Publishing, pp. 364-366.

Bordat A, Marchand G, Langlade NB, et al. 2017. Different genetic architectures underlie crop responses to the same pathogen: the \{helianthus annuus * phoma macdonaldii interaction case for black stem disease and premature ripening. BMC Plant Biol 17(1): 167.
Chen WM, Guo QY, Song HM, et al. 2008. Domestic new plant disease: The first report of the occurrence of sunflower Phoma black stem in Ili River Valley, Sinkiang, China. J Yunnan Agric Univ 23: 609-612 (in Chinese).

Darvishzadeh R, Sarrafi A. 2007. Genetic analysis of partial resistance to black stem (Phoma macdonaldii) in sunflower as measured by a seedling test. Plant Breed 126: 334-336.

Debaeke P, Pérès A. 2003. Influence of sunflower (Helianthus annuus L.) crop management on Phoma black stem (Phoma macdonaldii Boerema). Crop Prot 22: 741-752.

Larfeil C, Barrault G, Dechamp-Guillaume G. 2010. Assessment of sunflower genotype tolerance to Phoma macdonaldii. Oléagineux, Corps Gras, Lipides 17: 161-166.

Liu L, Li X. 1988. The geographical distribution of sunflower diseases in China. Plant Pathol 37: 470-474.

Luo J, Wu P, Liu Y, et al. 2011. Detection and identification of Phoma macdonaldii in sunflower seeds imported from Argentina. Australas Plant Path 40(5): 504-509.

Miric E, Aitken EAB, Goulter KC. 1999. Identification in Australia of the quarantine pathogen of sunflower Phoma macdonaldii (Teleomorph: Leptosphaeria lindquistii). Aust J Agric Res 50: 325-332.

Roustaee A, Costes S, Dechamp-Guillaume G, et al. 2000. Phenotypic variability of Leptosphaeria lindquistii (anamorph: Phoma macdonaldii), a fungal pathogen of sunflower. Plant Pathol 49(2): 227-234.

Seassau C, Debaeke P, Mestries E, et al. 2010. Evaluation of inoculation methods to reproduce sunflower premature ripening caused by Phoma macdonaldii. Plant Dis 94(12): 1398-1404.

Wu PS, Du HZ, Zhang XL, et al. 2012. Occurrence of Phoma macdonaldii, the causal agent of sunflower black stem disease, in sunflower fields in China. Plant Dis 96: 1696-1696.

Xu P, Han Y, Wu J, et al. 2007. Phylogenetic analysis of the sequences of rDNA Internal Transcribed Spacer (ITS) of Phytophthora sojae. J Genet Genom 34(2): 180-188.

Cite this article as: Yan N, Na R, Jia R, Zhang J, Zhao J. 2020. Occurrence of black stem on Helianthus annuus caused by Phoma macdonaldii and resistant evaluation of different sunflower varieties. OCL 27: 12. 